\title{
The Role of MANET in Collaborating IoT End Devices: A New Era of Smart Communication
}

\author{
https://doi.org/10.3991/ijim.v15i13.23045 \\ Vikram Narayandas $\left({ }^{凶}\right)$, M. Archana \\ Annamalai University, Chidambaram, India \\ narayandas.vikramegmail.com \\ D. Raman \\ Jawaharlal Nehru Technological University, Hyderabad, India
}

\begin{abstract}
Merging of MANET with IOT utilized in inevitable knowledge situations opens new possibility in checking and makes another communication stage for various applications in Internet of Things (IoT). Sensors utilized for IoT applications, sense nature and send the data to the gateway hub, which then send the congregated information to the MANET hub, particularly used for information gathering. MANET plays a key function, which is used as the foundation of the IoT arrange. Because of its intrinsic properties, the MANET hubs can assemble unconstrained associations with different hubs without the need of any base. MANET hubs can turn over the IoT procedure and accumulate information from the sensors and or any fixed Radiocommunication nodes. MANET cores can be utilized as key advances in a few applications in IoT. Because of the idea of self-designing, MANET hubs just as the sensor hubs can be sent in huge possibility. In this paper we have taken a survey of MANET and IOT various applications in running era and corelating various computing techniques like cloud, fog and edge with MANET and IOT. This examination is an improvement and usage of existing versatile specially selected procedure communication exploiting the in the structure of the IoT.
\end{abstract}

Keywords-MANET-IOT, Cloud, Edge computing

\section{Introduction}

The Mobile ad-hoc network (MANET) will play a vital job in the Internet of Things (IoT). The MANET is a type of inaccessible systems that have being self-ordering and auto connected in a reorganised framework. Each gadget in MANET can be moved obviously beginning with one zone then onto the following toward any way. They can make a system with their neighbours' shrewd gadgets and forward information to another gadget.Every MANET node works as a switch, as a system end structure and is firmly identified with WSNs. The connection among MANET and IoT opens new ways for administration arrangement in savvy situations and testing issues in its systems administration perspectives. One of the significant factors in MANET-IoT frameworks 
is the imperativeness changing over hubs, since the IoT system depends usually on various remote sensors and determination from MANET conventions centres around the most productive and briefest courses. An appropriate usage of sensor's battery power is a critical key in keeping up organize availability of a multi hop remote system. Remote approach rules cannot be utilized legitimately because of asset imperatives of sensors' cores, computational velocity, and human interface with hub's gadgets and system hub thickness. In this method, here is a need of composite answer for steering over MANET-IoT systems, which can utilize hub lingering vitality proficiently and broaden the system lifetime.[1].

a) Qualities of MANET

1. Dynamic Topologies: Network topology which is normally multihops, may change arbitrarily and quickly with time, it can shape unidirectional or bi-directional connections.

2. Data transmission compelled, variable limit joins: Wireless connections for the most part have lower unwavering quality, effectiveness, steadiness, and limit when contrasted with wired system. The throughput of remote correspondence is even not exactly a radio's most extreme transmission rate subsequent to managing the imperatives like numerous entrances, commotion, obstruction conditions, and so forth.

3. Self-sufficient behaviour: Each hub can go about as a host and switch, which shows its self-sufficient conduct.

4. Vitality constrained operation: As all the hubs differ on batteries or other superfluous methods for their vitality. Portable hubs are depicted with less memory, force and light weight highlights.

5. Restricted security: Wireless system are progressively inclined to security dangers. A brought together firewall is missing because of its circulated nature of activity for security, steering and host setup.

6. Less human intervention: They require least human mediation to arrange the system, subsequently they are powerfully independent in nature.

The Internet of Things (IoT) is a worldwide biological system of data and correspondence sequences got ready for interfacing any kind of thing, whenever and in anywhere, to one another and to the Internet. The application areas of IoT are assorted, spreading over from urban areas, building and home computerization, transportation and coordination's, and natural observing, to keen venture conditions, associated home machines, and shrewd wearable gadgets [2].In the up and coming age of computing, Mobile ad-hoc network (MANET) will take part in a significant job on the Internet of Things (IoT). The MANET is a sort of remote systems that are self-arranging and auto associated in a decentralized framework. Each gadget in MANET can be moved openly beginning with one area then onto the next in the direction of any path. They can make a system with their neighbour's keen gadgets and forward information to another gadget. 


\section{Literature Survey}

Deployment of IoT in a few areas like medical, home computerization, mechanical robotization, portable human services, power transmission and conveyance and so on. The figure 1 shows disposition of IOT in various fields.

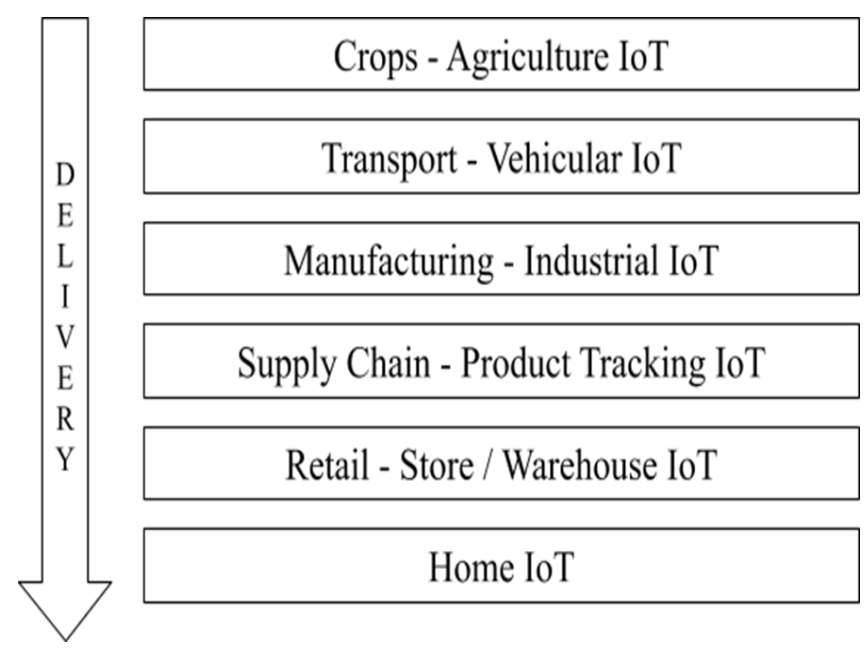

Fig. 1. IoT Solutions Delivery enablement from food to home fields

Presently urban areas are one of the developing spaces of IoT, where professionals are suggesting a few correspondence models and norms and give a few applications which encourage the dwelling residents by improving their ordinary standard of life. Sensor devices, which are exceptionally modest minimal effort gadgets, can detect various parameters in the earth, it can reserve information in its memory and can express with different hardware gadgets by utilizing correspondence innovation like IEEE802.15.4 or Bluetooth Low Energy (BLE). Then yet again RFID chip is additionally a valuable gadget in IoT, which is applied to distinguish an article appended with a RFID tag. [3]. Rather than interfacing the low force sensor hubs to the Internet legitimately, we have utilized MANET hubs in the core of the sensor and Internet Gateway. Sensor hubs or RFID empowered articles either send information to the MANET hub or to 'Access point" of WLAN and in the two cases information is sent by means of sensor passage hubs. There are a few applications in urban areas, where these gadgets assume a essential role and present various types of assistance to the community which incorporate checking of condition, the executives of traffic and so forth.Potential outcomes of wide use of IoT contexts in various regions are legitimately subject to the chances of interoperability between various communication advancements. The development of sensors prompts the expanding human requirement for remote observing of various processes. Essentially, WSN which comprises of various sensors that are proficient to peruse information from and store it and move them to another system hub, which is additionally a sensor. So the information, detected 
and moved from different sensors, are transmitted to the main hub, which is generally called sink [24]. Remote sensor organizes fundamentally are the essential component in the worldwide IoT framework, as sensors can assemble data from various things and transmit it over the system. Not with standing, the constancy of IoT contexts is profoundly reliant on the influence utilization and versatility of WSN [25]. Numerous regularization bodies have grown new conventions and structures for the IoT, among them one is IETF. The IETF (Internet Engineering Task Force) plays a huge job in characterizing new measures for the IoT. The most broadly utilized measures characterized by the IETF is Constrained Application Protocol (CoAP) which is a specific Internet Application Protocol expected for use in asset obliged Internet gadgets [26], IPv6 over Low-Power Wireless Personal Area Networks (6LoWPAN). which gives a system to suit IPv6 information correspondences on IEEE 802.15.4 systems [27], and the IPv6 Routing Protocol for Low-Power and Lossy Networks (RPL) [28] which, is the IPv6 Routing Protocol for required systems. IETF has created other IoT principles at the application layer, including another help revelation for the IoT and new serializations, for example, the Constrained RESTful Environments (CoRE) Link Format [29].Current improvement in Mobile Adhoc Networks (MANET) which helps enthusiastically development of system and cooperating without predefined arrangement shows incredible triumph joining various IoT based introduction area in keen towns. Pervasive Computing and Internet of Things are very famous in late age and along these lines bestowing elevated level security component is exceptionally crucial for such propelled specialized frameworks. To make IoT a reality for keen condition, progressively alluring to end clients, and financially fruitful, it must be perfect with WSNs and MANETs

\section{Integrating IoT End Devices With Manet}

Associated gadgets, for example, shrewd home robotization portal, keen forced air systems, savvy centre points, brilliant indoor regulator, shading changing shrewd lights, shrewd cell phones, keen watches and brilliant tablets, and so forth are ubiquitous in our regular daily existences and are turning into a significant apparatus with remote systems administration highlights utilizing diverse remote conventions normally utilized. Routes permit connections between clients inside an Internet of Things environment framework. These keen gadgets are consequently associated, and a system is shaped without anyone else. In any case, there are numerous difficulties all through this built up system of its own for secure correspondence. Security has been a mainstream hindrance to receiving the cloud model of web authenticity. The capability and resource the executives might be in the cloud condition is a diffused structure that puts the world in a raised circumstance with numerous worries over its shortcomings, security dangers and challenges. Distinctive investment parties have widened those issues relying upon the perspective and objective of each gathering.

MANET may work as independent style, or they can be the piece of wider web. They structure exceptionally powerful self-ruling topology with the closeness of one or various distinctive handsets between hubs. The standard task for the MANET is to 
prepare every gadget to persistently maintain ahead the data required to appropriately course traffic. MANETs comprise of a distributed, self-shaping, self-retrieving system MANET's around 2000-2015 commonly impart at radio frequencies (30MHz-5GHz). This can be utilized in street wellbeing, extending from sensors for condition, home, health, calamity salvage activities, air/land/naval force protections, weapons, robots, and so on.At present, IoT is quickly growing and has driven the excited advancement of different related application administrations. It has been applied in numerous fields, for example, keen home [15], human wellbeing identification, catastrophe the executives, building structure security, individual distinguishing proof [16][17][18], etc.

\subsection{Role of MANET in IoT}

Disastrous occasions are one of the most testing utilizations of multi-hop adhoc systems because of potential harms of existing media transmission framework. The conveyed cell correspondence framework may be incompletely or totally pulverized after a cataclysmic event. Multi-hop ad hoc transmission is a intriguing choice to manage the absence of interchanges in a disaster situation. They have advanced since their source, prompting distinctive especially different ad hoc models, for example, MANETs, VANETs, DTNs, or WSNs. The vision of the Internet of Things (IoT) is at introduce motivating analysis in various zones [9] Universal detecting and inevitable processing in IoT depend on the communication framework gave by remote specially appointed systems (MANET). The figure 2 shows how nodes passes information from one node to other in MANET-IOT disposition.

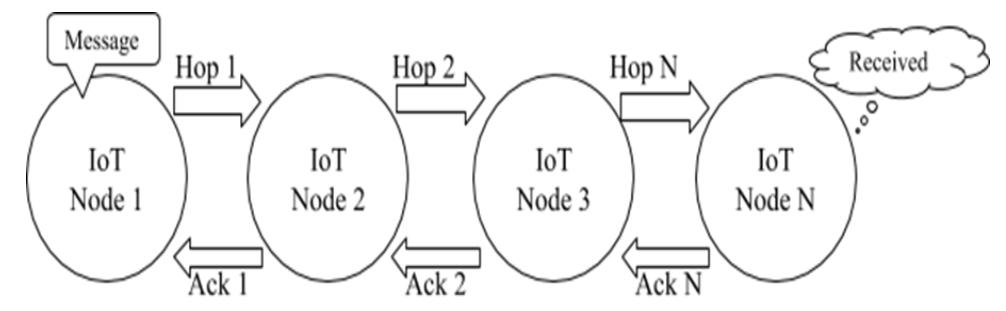

Fig. 2. Basic MANET IoT Nodes integrating for successfully transferring a message

These are decentralized and give an all-around organized information scattering design after sending. There occurs an assortment of availability alternatives for the IoT, for example, Bluetooth, WiFi, Cellular, and ZigBee. The last depends on the IEEE 802.15.4 norm, which has critical favourable situations in complex frameworks offering low-power activity and high adaptability. IoT applications require a consistent reconciliation into information mists by Internet advancements. 6LoWPAN is a systems administration adjustment layer that permits IPv6 bundles to be conveyed productively inside little connection layer outlines, as characterized by IEEE 802.15.4. While the correspondence stack for IoT is prepared up to the system layer, the structure of the information spread layer is as yet an open issue [10]. 
Favourable circumstances and Dis-advantages of interest of incorporating MANET and IoT:

a) Favourable circumstances:

1. Division from core system organization.

2. Every hub can assume both the jobs for example of router or switch and host indicating self-governing nature.

3. Self-designing and self-improving hubs doesn't require human intercession.

b) Dis-advantages:

1. Assets are restricted because of different limitations like commotion, impedance conditions, and so forth.

2. Absence of approval functions.

3. Progressively inclined to assaults because of restricted physical security.

\subsection{Associating cloud-MANET with IoT}

The IoT Cloud-MANET system of keen gadgets is made out of IoT, distributed computing, and MANET. This structure can get to and convey cloud administrations to the MANET clients through their shrewd gadgets in the IoT system where all calculations, information taking care of, and asset the board are performed. The gadgets can move beginning with one region then against the following inside the scope of the MANET arrange. Different MANETs can associate with a similar cloud, they can utilize cloud administration in an ongoing. For associating the savvy gadget of MANET to cloud needs incorporation with versatile applications [4].

Verge of the cloud: Cloud computing offers a stack of advantages for end clients: top of the line machines, mind overwhelming measures of capacity, high accessibility, and everything accessible at the bit of a catch. Clouds that do not just offer stockpiling yet additionally calculations that can be re-appropriated in type of virtual machines (VMs). In the most adaptable and general cloud computing model ("Infrastructure as-a Service", IaaS), clients can run whole Virtual Machines (VMs) inside the Cloud. VM pictures work as formats from which a basically vast number of VM occurrences can be started up. Foundation as-a-Service (IaaS), gives Virtual Machines (VMs) completely fulfilling the client needs as much as assets. The assets of the providers are normally facilitated as a server farm. A service provider ought to have conveyed server farms all around the globe to offer types of assist to the clients

\section{Advantages}

a) No forthcoming venture: Cloud registering utilizes a pay as you-go evaluating model. A specialist co-op does not have to put resources into the framework to begin picking up advantage from cloud computing. It just leases assets from the cloud as per its own needs and pay for the utilization.

b) Lowering working cost: Resources in a cloud domain can be quickly dispensed and de-distributed on request. Henceforth, a specialist organization no longer needs to 
arrangement limits as per the pinnacle load. This gives tremendous investment funds since resources can be discharged to save money on working costs when administration request is low.

c) Highly versatile: Infrastructure suppliers pool enormous measure of resources from server farms and make them effectively available. A service provider can without much of a stretch grow its support of enormous scopes to deal with fast increment in administration requests (e.g., streak swarm impact). This model is occasionally called surge processing.

Clouds have practically boundless abilities as far as capacity and planning power. The requirement for huge scope IoT applications has set off the building of the IoT and distributed computing ideal models, using the adaptability, execution, and pay-more only as costs arise abilities of the Cloud and making up for the mechanical imperatives of IoT frameworks [5].

Attributes of the Cloud Essential for IoT:

1. On-request self-administration

2. Broad network gain access to

3. Resource pooling

4. Location autonomy

5. Rapid versatility

6. Measured service

Merging IoT: Designing applications for IoT and Cloud is tricky for various reasons; even without thinking about the intrinsic comprehensive nature of distributed registering, there are a few challenging stages, programming styles and correspondence rules. It tends to be contended this is keeping down the enterprise all in all: Applications are hard to compose, convey and oversee. In the constant handling, another rising innovation where the need of associating keen gadgets with cloud through Internet has raised. IoT gadgets handled data is to be put away and gotten to anyplace required with a help of ground-exceeding registering execution, proficient capacity foundation for heterogeneous frameworks and programming which arranges and controls these a variety of appliances. A ton of difficulties to be inclined to are recorded with this new rising innovation as it should be perfect with the up-and-coming $5 \mathrm{G}$ remote gadgets as well. Convergence of IoT frameworks and the Cloud activities is their capacity to stream data to the Cloud in an adaptable and best way, while simultaneously providing the way to overseeing applications and communication streams and giving information driven IoT administrations at scale. These efforts are profoundly helpful and divine well for applications with countless IoT gadgets, spread potentially across immense geographical zones. In such a building up, the Cloud will give a united and joined stage for quick, adaptable, and productive advancement of end-client applications [4,6,7].

Incorporating smart gadgets: First there was PC, at that point came cell phone and now there are numerous gadgets that individuals use as figuring stages. Tablets, TVs, eBook per users are artifacts of days gone by. Presently there are Google Glass, Samsung Watch, Smart TV and some more. The rundown of gadgets with processor for calculations that can be associated with Internet are expanding each day. Organizations 
are picking to utilize shrewd gadgets to expand quality yield. Following are two or three fascinating employments of Google Glass-

a) Fast preparing for representatives: It takes a great deal of preparing to work a laser shaper. This can be shown more proficiently by overlaying visual guides onto the machine, empowering workers to figure out how to utilize the gear quicker than traditional instructional exercises.

b) Museum tours: Audio accounts utilized as of now will be upgraded with noticeable components. It would be incredible to examine at any artwork hanging at the Met, have a product to remember it and recover extra data on request by a basic signal.

c) Cloud computing has become an incredible empowering influence of cross stage applications, for example applications that can run on different stages.

\section{Advances behind cross platform cloud applications:}

1. Cross platform: This is a typical system that empowers a similar programming code to get executed on various stages.

2. Cloud applications: In Cloud Application, most or all the information is put away on remote servers and conveyed over a system.

3. Software: The normal system is facilitated inside the cloud itself with insignificant programming as required by the client's gadget. By and large, the interface is electronic, with just fundamental Internet availability being the customer's necessity. In this way a cross stage cloud application is consistently compact. If one server closes, the administration proceeds without getting saw by end clients. If the hardware changes, the upgraded one is incorporated to the aggregate pool of assets so the product can adjust to interface with the new gear.

Hardware Equipment innovation and IT industry are experiencing significant changes. Particularly the ones identified with arrange availability are increasing new standards. Subsequently organizations would prefer not to secure their product in restrictive frameworks however are somewhat picking to structure their data conveyance process around models that are free of the equipment they run on.The smart gadget to gadget correspondence in the cloud-MANET structure of the IOT of things is a innovative strategy that finds and associated close by keen gadgets with no incorporated framework [8]. The proposed procedure will be helpful in machine to machine (M2M) systems since, in the M2M arrange, there are a few gadgets close by to one another. The shrewd gadget clients will utilize cloud administration to find the gadgets, edge helpful data in enormous information and can process recordings, pictures, content, and sound.

\subsection{Intermediating fog}

Fog-based architecture design that empowers secure nearby correspondences among IoT gadgets. The arrangement connects the adaptability and security of cloud based IoT organizations with the flexibility and asset of fog considering. Existing recommendations accept that the fog organize is liable for building up secure 
correspondences among things and that each fog hub is trusted and secure against outside assailants [11] Fog hubs are parts that can be learned to buy things without requiring Internet network. They provide things with extra administrations and assets that in conventional cloud-helped designs are actualized by cloud administrations. Haze hubs can be actualized as physical or virtual frameworks over heterogeneous registering gadgets, going from low-power PCs to bunch of ground-breaking server machines. Models incorporate propelled arrange passages dependent on medium-power ARM CPUs and smaller than usual server farms for exceptionally accessible capacity and information analytics [12][13]. The figure 3 shows how cloud computing, fog and edge computing associating with IOT end devices.

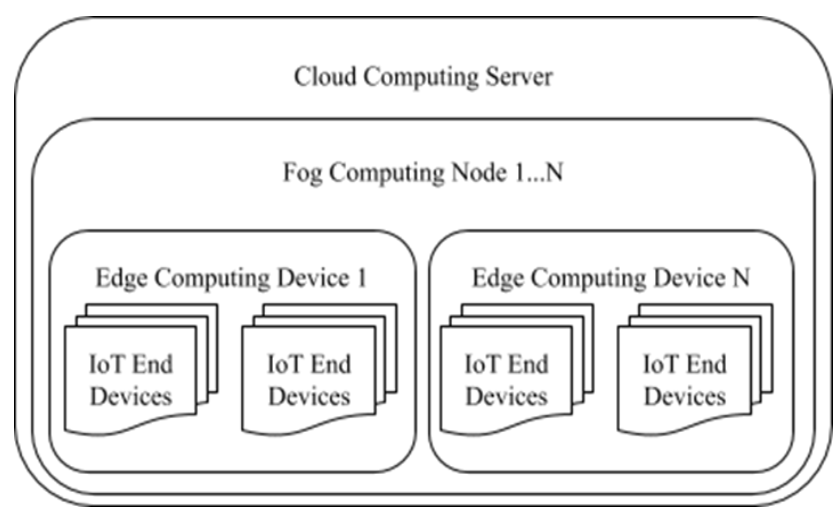

Fig. 3. Corelating Cloud, Fog with Edge and IOT end devices

To set up secure correspondences, all things, fog hubs, and cloud administrations should commonly confirm with one another by utilizing solid qualifications, for example, symmetric cryptographic keys. We expect that cloud administrations go about as personality suppliers and foundations of trust to manufacture a versatile confirmed framework and that distinctive cloud administrations can utilize character alliance frameworks to empower cross-validation among their separate things and administrations Fog hubs don't have severe equipment limitations and can commonly verify against cloud benefits by utilizing TLS or DTLS protocols [14].

\subsection{Enhancing the edge}

Edge computing gives organize, processing, application, and capacity administrations to close clients through disseminated edge hubs. Edge computing can advance issues, for example, postponement and availability by offloading some portion of the computational assignments to edge devices near the information source. Utilizing this element, IoT nodes can spare more assets while as yet keeping up the nature of administration. Notwithstanding since calculation offloading choices concern joint and complex asset the executives [19]. The figure 5 represents the role of Edge Computing Devices in enabling IoT. 


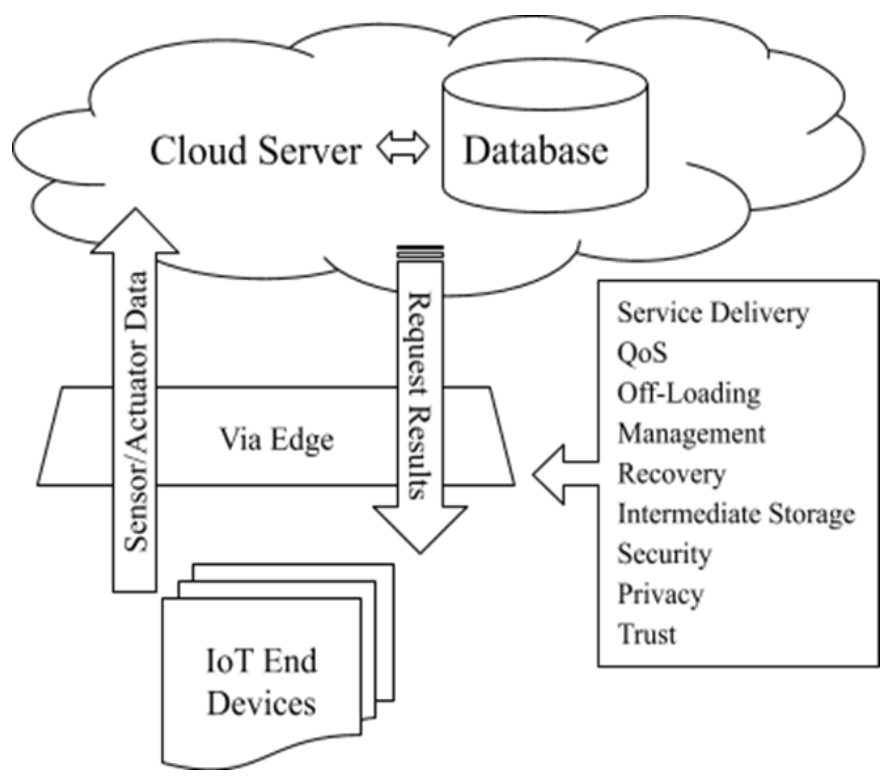

Fig. 4. Role of Edge Computing Devices in enabling IoT

The end gadgets in edge registering are assorted, for example, associated vehicles, keen cameras [22] [23], and so forth, and they are makers of information and errands with information pre-handling and information transmission capacities. In any case, when an end gadget needs to deal with an errand with an enormously huge computational asset, it is regularly hard to depend on the registering capacity of the gadget itself to fulfil the need. Subsequently, it very well may be understood by edge processing utilizing the figuring assets of the edge hub. The edge hub is geologically near the end gadget and can give excellent system association and figuring administrations. Contrasted and the end gadget, the edge hub has a more impressive figuring ability to process the errand, and the edge hub reacts quicker to the end gadget than the cloud. In this way, by utilizing edge hubs to play out some computational undertakings, the reaction speed of the errand can be improved while guaranteeing exactness. Likewise, the edge hub additionally has a storing capacity, which can abbreviate the reaction time of re-access by reserving objects with high access heat.[20] [21].

\section{Conclusion}

Looking into a great deal of how MANET systems work in IoT and which are its focal points and impediments to the end that this sort of systems could help individuals by and large in basic circumstances. The Ad-hoc mode can be utilized by applications where some sort of systems has more end devices for the overall population. The MANET systems among savvy gadgets in IoT is being tried and executed. Thus, future work will in general spotlight on meeting MANET, WSN, and LTE, as an attempting 
to inspect the capacity to expand the heterogeneity of increasingly various measures by attempting to associate the sink hub that deals with IEEE 802.11 to a LTE gadget. On the other hand, future work can include how to expand the inclusion territory of MANET hubs by including LTE innovation in the correspondence to permit everything associated with the web to converse with one another, and to take the most extreme conceivable favourable position. Despite the actuality that the formation of communication between the intuitive gadgets in a heterogeneous structure of $5 \mathrm{G}$ system would be trying on a similar line, the spending plan of Cloud-MANET structure will provide the resolution for the designers

\section{$5 \quad$ References}

[1] Kamel, Mohammed B. \& Alameri, Ibrahim \& Onaizah, Ameer. (2017). STAODV: A secure and trust-based approach to mitigate blackhole attack on AODV based MANET. https://doi.org/10.1109/iaeac.2017.8054219

[2] LuigiAtzori, AntonioIera, and Giacomo Morabito. 2010. The internet of things: Asurvey. Computer Networks 54, 15 (2010), 2787-2805.

[3] Networking for IoT and applications using existing communication technology Egyptian Informatics Journal Volume, July 2018, Pages 107-127.

[4] Alam, Tanweer \& Benaida, Mohamed. (2018). The role of cloud-MANET framework in the Internet of Things (IoT). International Journal of Online Engineering (iJOE). 14. 97-111. https://doi.org/10.3991/ijoe.v14i12.8338

[5] Alessio Botta, Walter de Donato, Valerio Persico, and Antonio Pescapé. 2014. On the integration of cloud computing and Internet of Things. In Proc. of the 2014 Int. Conference on Future Internet of Things and Cloud (FICLOUD'14). Washington, DC, 8. https://doi.org/10.1109/ficloud.2014.14

[6] IBM Internet of Things Foundation. http://internetofthings.ibmcloud.com.

[7] "Context-Driven and Real-Time Provisioning of Data-Centric IoT Services in the Cloud" ACM Transactions on Internet Technology, Vol. 19, No. 1, Article 7. Publication date: November 2018.

[8] Pelusi, A. Passarella, and M. Conti. 2006. Opportunistic networking: data forwarding in disconnected mobile ad hoc networks. Comm. Mag. 44, 11 (November 2006), 134-141. https://doi.org/10.1109/mcom.2006.248176

[9] Whitmore, A. Agarwal, and Li Da Xu. 2015. The internet of things-A survey of topics and trends. Inf. Syst. Front. 17, 2 (01 Apr. 2015), 261-274. https://doi.org/10.1007/s10796-014$\underline{9489-2}$

[10] Akerman's, R. Bachiller, N. Matthys, W. Joosen, D. Hughes, and M. Vuc ‘inić. 2016. Towards efficient publish-subscribe middleware in the IoT with IPv6 multicast. In Proceedings of the 2016 IEEE International Conference on Communications (ICC).https://doi.org/10. $\underline{1109 / \text { icc. } 2016.7511254}$

[11] Carlo Puliafito, Enzo Mingozzi, Francesco Longo, Antonio Puliafito, and Omer Rana. 2019. Fog Computing for the Internet of Things: A Survey. ACM Trans. Internet Technol. 19, 2 , Article 18 (April 2019), 41 pages. https://doi.org/10.1145/3301443

[12] Paolo Bellavista and Alessandro Zanni. 2017. Feasibility of fog computing deployment based on docker containerization over RaspberryPi. In Proceedings of the ACM 18th International Conference on Distributed Computing and Networking. https://doi.org/10. $\underline{1145 / 3007748.3007777}$ 
[13] FrankWang, JamesMickens, Nickolai Zeldovich, and Vinod Vaikuntanathan. 2016. Sieve: Cryptographically enforced access control for user data in untrusted clouds. In Proceedings of the 12th USENIX Symposium on Networked Systems Design and Implementation.

[14] Luca Ferretti, Mirco Marchetti, and Michele Colajanni. 2019. Fog-based Secure Communications for Lowpower IoT Devices. ACM Trans. Internet Technol. 19, 2, Article 27 (March 2019), 21 pages. https://doi.org/10.1145/3284554

[15] Yin Jie, Ji Yong Pei, Li Jun, Guo Yun, and Xu Wei. 2013. Smart home system based on IoT technologies. In Proceedings of the 2013 International Conference on Computational and Information Sciences. IEEE, 1789-1791. https://doi.org/10.1109/iccis.2013.468

[16] Xiaoya Hu, Bingwen Wang, and Han Ji. 2013. A wireless sensor network-based structural health monitoring system for highway bridges. Computer-Aided Civil and Infrastructure Engineering 28, 3 (2013), 193-209. https://doi.org/10.1111/j.1467-8667.2012.00781.x

[17] S.M. Riazul Islam, Daehan Kwak, M.D. Humaun Kabir, Mahmud Hossain, and Kyung-Sup Kwak. 2015. The Internet of Things for health care: A comprehensive survey. IEEE Access 3 (2015), 678-708. https://doi.org/10.1109/access.2015.2437951

[18] M. D. Kamruzzaman, Nurul I. Sarkar, Jairo Gutierrez, and Sayan Kumar Ray. 2017. A study of IoT-based post-disaster management. In Proceedings of the 2017 International Conference on Information Networking (ICOIN). IEEE, 406-410. https://doi.org/10.1109/ icoin.2017.7899468

[19] Shihao Shen, Yiwen Han, Xiaofei Wang, and Yan Wang. 2019. Computation Offloading with Multiple Agents in Edge-Computing-Supported IoT. ACM Trans. Sen. Netw. 16, 1, Article 8 (December 2019), 27 pages. https://doi.org/10.1145/3372025

[20] ETSI.2014.MobileEdgeComputingIntroductoryTechnicalWhitePaper.https://portal.etsi.org /Potals/0/TBpages/MEC/Docs/Mobileedge_Computing_Introductory_Technical_White_Pa per V1\%2018-09-14.pdf.

[21] Nasir Abbas, Yan Zhang, Amir Taherkordi, and Tor Skeie. 2017. Mobile edge computing: A survey. IEEE Internet of Things Journal 5, 1 (2017), 450-465. https://doi.org/10.1109/ jiot.2017.2750180

[22] Dennis Grewe, Marco Wagner, Mayutan Arumaithurai, Ioannis Psaras, and Dirk Kutscher. 2017. Information-Centric Mobile Edge Computing for Connected Vehicle Environments: Challenges and Research Directions. In Proceedings of the Workshop on Mobile Edge Communications (MECOMM '17). Association for Computing Machinery, New York, NY, USA, 7-12. https://doi.org/10.1145/3098208.3098210

[23] Ganesh Ananthanarayanan, Victor Bahl, Landon P. Cox, Alex Crown, Shadi Nogbahi, Yuanchao Shu:Video Analytics - Killer App for Edge Computing. MobiSys 2019: 695-696. https://doi.org/10.1145/3307334.3328589

[24] P. Bellavista, G. Cardone, A. Corradi, L. Foschini, "Convergence of MANET and WSN in IoT Urban Scenarios”, IEEE Sensors Journal, Vol. 13, No. 10, pp. 3558-3567, 2013. https://doi.org/10.1109/jsen.2013.2272099

[25] Gregory S. Yovanof and George N. Hazapis. 2009. An Architectural Framework and Enabling Wireless Technologies for Digital Cities \& Intelligent Urban Environments. Wirel. Pers. Commun. 49, 3 (May 2009), 445-463. https://doi.org/10.1007/s11277-009-9693-4

[26] Z. Shelby, K. Hartke, and C. Bormann. 2014. The Constrained Application Protocol (CoAP). RFC 7252. Retrieved January 11, 2020 from https://doi.org/10.17487/rfc7252

[27] Ishaq, Isam \& Carels, David \& Teklemariam, Girum \& Hoebeke, Jeroen \& Van den Abeele, Floris \& De Poorter, Eli \& Moerman, Ingrid \& Demeester, Piet. (2013). IETF Standardization in the Field of the Internet of Things (IoT): A survey. Journal of Sensor and ActuatorNetworks.2.235-287.https://doi.org/10.3390/jsan2020235 
[28] T. Winter (Ed.), P. Thubert (Ed.), A. Brandt, J. Hui, R. Kelsey, P. Levis, K. Pister, R. Struik, J. P. Vasseur, and R. Alexander. 2012. RPL: IPv6 Routing Protocol for Low-Power and Lossy Networks. RFC 6550 (Proposed Standard). Retrieved January 11, 202 from https://doi.org/10.17487/rfc6550

[29] Z. Shelby. 2012. Constrained RESTful Environments (CoRE) Link Format. RFC 6690. Retrieved January 11, 2020 from https://doi.org/10.17487/rfc6690

\section{Authors}

Vikram Narayandas, Research Scholar in Department of Information Technology, Annamalai University, Chidambaram, Tamil Nadu, India. And Member of CSI, IAENG and published 9 international journals. Email: narayandas.vikram@gmail.com

Dr. Maruthavanan Archana, presently working as Assistant Professor in Department of Information Technology, Faculty of Engineering and Technology, Annamalai University, Chidambaram, Tamil Nadu, India. And Member of CSI, IAENG and published 12 international journals.

Dr.D.Raman, working as Professor in Department of Computer Science and Engineering, Varadaman College of Engineering, Hyderabad, India. And member of CSI, IAENG and senior member of IEEE, published 19 international journals.

Article submitted 2021-04-02. Resubmitted 2021-05-13. Final acceptance 2021-05-15. Final version published as submitted by the authors. 